\title{
Effects of Ground Constituent Parameters on Array Mutual Coupling for DOA Estimation
}

\author{
Irfan Ahmed, Warren F. Perger, and Seyed A. Zekavat \\ Department of Electrical and Computer Engineering, Michigan Technological University, 1400 Townsend Drive, Houghton, \\ MI 49931, USA \\ Correspondence should be addressed to Irfan Ahmed, iahmed@mtu.edu
}

Received 12 May 2011; Revised 28 July 2011; Accepted 3 August 2011

Academic Editor: Hoi Shun Lui

Copyright ( 92011 Irfan Ahmed et al. This is an open access article distributed under the Creative Commons Attribution License, which permits unrestricted use, distribution, and reproduction in any medium, provided the original work is properly cited.

Effects of ground constituent parameters on the mutual coupling (MC) of monopole antenna array are investigated. This work augments an existing $\mathrm{MC}$ compensation technique for ground-based antennas and proposes reduction in mutual coupling for antennas over finite ground as compared to the perfect ground. The work is investigated by finite element method analysis, and numerical results are presented. A factor of 4 decrease in both the real and imaginary parts of the mutual coupling is observed when considering a poor ground versus a perfectly conducting one, for quarter-wave monopoles in receiving mode. A simulated result to show the errors in direction-of-arrival (DOA) estimation with actual realization of the environment is also presented.

\section{Introduction}

There is an emerging trend in wireless applications such as safety and security, command, and control MIMO communication, that require antennas with direction-of-arrival (DOA) and beamforming capability. In order to support DOA estimation and beamforming, antenna arrays are used. Traditionally, antenna arrays consist of closely located antenna elements that are uniformly distributed across the array. To determine DOA, several techniques have been developed [1]. These techniques frequently assume that the sensors are ideal and operate in an isolated environment. In practice, however, this is not true. The real antenna elements not only interact with each other (MC) but also with the surroundings. This results in the distortion of the signal and causes error in DOA estimation.

Several techniques have been proposed to overcome the errors due to antenna MC. These techniques are in the process of development, but can be classified in terms of autocalibration [2-4], open circuit voltage method [5], numerical techniques [6-8], offline calibration [9], and receiving mutual impedance methods [10]. In general, these techniques do not consider the interaction of the antenna array with an imperfect ground in the near zone [11]. Effects of ground proximity and constituent parameters on wire antennas have been presented in the literature [12-14].

This paper considers the effects of ground on the MC of an array of monopoles and impact on DOA estimation. Monopole antennas that take advantage of image theory are ideally placed above a perfect ground of conductivity $\sigma=\infty$ and relative permittivity $\epsilon_{r}=1$. The real ground or earth has finite conductivity and may have high permittivity. The effects of ground constituent parameters on $\mathrm{MC}$ and ultimately on DOA estimation are investigated. The evaluation of $\mathrm{MC}$ is an extension of the technique that finds $\mathrm{MC}$ of a monopole array over a high conductivity ground $\left(\sigma \cong 10^{7}, \epsilon_{o}, \mu_{o}\right)[10]$ to a finite ground $\left(\sigma \leq 1, \epsilon, \mu_{o}\right)$. The newly found MC is used to compensate error in DOA estimation for an array over imperfect ground.

\section{Problem Formulation}

Consider a uniform linear array (ULA) of $\mathrm{M}$ omnidirectional elements. Suppose that plane waves from $J$ narrowband far-field sources are incident on this array and $M>J$. 
The azimuth directions of uncorrelated incident signals are $\phi_{1}, \phi_{2}, \ldots, \phi_{J}$. The $k$ th sample of the array output is given as

$$
\mathbf{X}[k]=\mathbf{U}[k]+\mathbf{N}[k]
$$

where $\mathbf{X}[k]=\left[x_{1}[k], x_{2}[k], \ldots, x_{M}[k]\right]^{T}, \mathbf{U}[k]=\left[u_{1}[k]\right.$, $\left.u_{2}[k], \ldots, u_{M}[k]\right]^{T}$ is the coupling-free voltage at the antenna terminal, and $\mathbf{N}[k]=\left[n_{1}[k], n_{2}[k], \ldots, n_{M}[k]\right]^{T}$ is a vector of white Gaussian noise samples appearing at the antenna terminal receiver. The noise is zero mean and has correlation matrix $\sigma^{2} \mathbf{I}$ where $\sigma$ is the standard deviation and $\mathbf{I}$ is an $M \times$ $M$ identity matrix. The signal model in (1) does not consider mutual coupling within the array. Elements in a real antenna array interact with one another due to mutual coupling. The relation between actual voltage $\mathbf{V}$ at the antenna terminal and theoretical coupling-free voltage $\mathbf{U}$ is given as

$$
\mathrm{ZV}=\mathrm{U}
$$

where $\mathbf{Z}$ is an $M \times M$ matrix whose coefficients can be determined by electromagnetic analysis such as MoM or finite element method FEM. Thus, a more accurate signal model for the array output (1) is given as:

$$
\mathbf{X}[k]=\mathbf{Z V}[k]+\mathbf{N}[k] .
$$

The zero mean Gaussian noise in the receiver and signal generation in the far-field sources are independent processes. Therefore, the spatial covariance matrix for (3) is given as

$$
\mathbf{R}=E\left\{\mathbf{Z} \mathbf{V} \mathbf{V}^{\prime} \mathbf{Z}^{\prime}\right\}+\sigma^{2} \mathbf{I},
$$

where $\sigma^{2}$ is the noise variance and $\mathbf{I}$ is the identity matrix. It is evident from (4) that correct knowledge of $\mathbf{Z}$ is pivotal in DOA estimation with minimum error. Considering array elements as monopoles, a method to find $\mathrm{Z}$ as the receiving mode mutual impedance is in the literature [10]. This method considers a monopole antenna over a high conductivity or perfect ground. However, in real-world applications an antenna may be placed over a finite or low conductivity ground.

Consider an array of two $\lambda / 4$ monopole antenna at $2.4 \mathrm{GHz}$ over an arbitrary ground as shown in Figure 1. The array is excited by an incident plane wave and each element is connected to a load $Z_{L}$. Due to this excitation, terminal currents $I_{1}^{t}, I_{2}^{t}$ flows in the loads of antenna no. 1 and no. 2, respectively. The terminal voltage at antenna terminal no. 1 is given as

$$
V_{1}^{t}=I_{1}^{t} Z_{L}=U_{1}^{t}+W_{1}^{t}
$$

where $U_{1}^{t}$ is the voltage due the plane wave incidence alone and $W_{1}^{t}$ is the induced voltage due the flow of current in antenna no. 2; both causes are independent of one another. Therefore,

$$
W_{1}^{t}=I_{2}^{t} Z_{12}
$$

where $Z_{12}$ is the mutual impedance between element no. 1 and no. 2 due to the current in the load across port no. 2 .

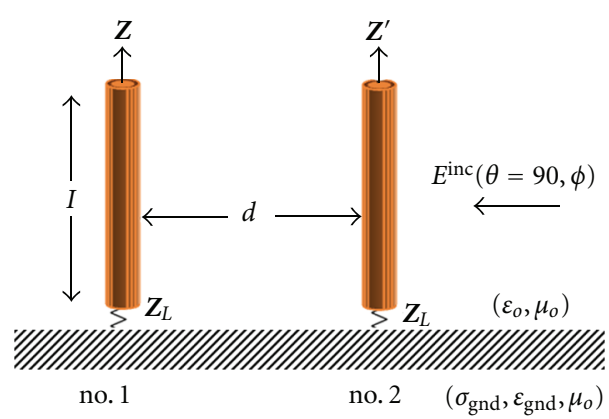

Figure 1: Setup for finding mutual coupling between monopole array over arbitrary ground.

Exploiting the principle of superposition, the current distribution $I_{1}$ along antenna no. 1 can be given as

$$
I_{1}=I_{1 U}+I_{1 W},
$$

where subscripts $U$ and $W$ correspond to the cause of the current distribution.

Therefore, the induced voltage $W_{1}^{t}$ is given as [15]:

$$
W_{1}^{t}=-\frac{1}{I_{1}^{t}} \int_{0}^{l} E_{z 12}\left(z^{\prime}\right) I_{1 w}\left(z^{\prime}\right) d z^{\prime},
$$

where $E_{z 12}\left(z^{\prime}\right)$ is the $E$-field component radiated by antenna no. 2 towards antenna no. 1 and $I_{1 w}\left(z^{\prime}\right)$ is the current distribution along antenna no. 1 . Hence, the mutual impedance in (5) can be given as

$$
Z_{12}=\frac{W_{1}}{I_{2}^{t}}=-\frac{1}{I_{2}^{t} I_{1}^{t}} \int_{0}^{l} E_{z 12}\left(z^{\prime}\right) I_{1 w}\left(z^{\prime}\right) d z^{\prime} .
$$

It is evident from (9) that, for a given current $I_{2}^{t}$, the $E$-field $E_{z 12}\left(z^{\prime}\right)$ has a major contribution in the numerical value of mutual impedance. Ideally, over a perfect ground the monopole antenna radiates strongly along the horizontal direction $\theta=90^{\circ}$, that is, towards the adjacent element in our configuration. This results in strong mutual coupling or sharing of energy between array elements. However, over a finite ground, the field strength in the horizontal direction is much smaller in the near field and almost zero in the far field [12, Chapter 23]. This reduction in field strength should reduce the mutual coupling between antenna elements placed over finite ground.

In the next section, we will investigate this hypothesis through the FEM and present results of mutual coupling by extending the existing technique [10] to the case of finite ground and its effects on mutual coupling and DOA.

\section{Results and Discussion}

The investigation of our hypothesis is carried out by considering two $\lambda / 4$ monopole antennas at $2.4 \mathrm{GHz}$ as shown in Figure 1 . The antennas are placed over an arbitrary ground with element spacing $\lambda / 2$ and are connected to a load $Z_{L}=$ $50 \Omega$. The array is excited by plane wave, whose incident 
direction is $\left(\theta=90^{\circ}, \phi=90^{\circ}\right)$. However, due to the axial symmetry of the antenna, this analysis is independent of incident azimuth direction $(\phi)$ for a given elevation $(\theta)$.

The mutual coupling found in [10] over a wide frequency range is compared for the case when the ground plane becomes poor as given in Table 1; see Figure 2. The result clearly shows that around the resonant frequency both the real and imaginary parts of $Z_{12}$ over poor ground reduce to about one-third of the value of $Z_{12}$ over perfect ground. It is also observed that mutual coupling undergoes very small variations over poor ground for a wide range of frequencies. This reduction of mutual impedance was motivation to investigate a wide variety of ground conditions usually encountered in wireless communication. Figure 3 shows that even for good electrical ground, usually made available for fixed antenna locations, the mutual coupling is still half of the value at perfect ground. This result is expected from the fact that over dielectric ground the monopole radiation becomes minimal along the horizontal direction and reduces the value of $W_{1}$ in (9). The result is consistent with the findings of $[12,13]$.

The variation of mutual impedance over a wide range of conductivity for a nominal relative permittivity $\epsilon_{r}$ is shown in Figure 4. It can be further deduced from the results that when the skin depth of the ground increases or the loss tangent decreases, the mutual impedance decreases and vice versa. We can also conclude that mutual coupling can be approximately divided between two ranges for which it assumes fairly constant values. These two ranges are when the loss tangent $\sigma / \omega \epsilon \leq 1$ or $\sigma / \omega \epsilon \gg 1$.

It is well known that soil water contents vary from place to place, and this may result in a change of permittivity of the ground. However, this change of water content will not cause any deviation in conductivity values over a wide range of frequencies [17]. The behavior of mutual impedance for such situations is investigated and results are shown in Figure 5. The curves account for variation from dry land to a saline medium such as sea water whose $\epsilon_{r}<100$. The result depicts that, for a good ground, the increase in relative permittivity increases the mutual impedance.

Investigation of the effects of arbitrary ground mutual coupling on DOA estimation is carried out by simulation. An array of five vertical quarter-wave monopoles is taken as DOA estimator antenna, the spacing between elements is $\lambda / 2$ and it is operating at $2.4 \mathrm{GHz}$. The mutual coupling matrix $\mathbf{Z}$ is calculated according to [10] by placing antennas over poor and perfect ground, respectively. The mutual impedances are given in Table 2 whose second column contains values from [18]. DOA estimation is carried out for a vertically polarized far field source at $\theta=90^{\circ}, \phi=90^{\circ}$. The terminal voltage vector $\mathbf{V}$ at antenna ports is measured in COMSOL multiphysics environment [19], for a terminal load of $50 \Omega$. The effect of mutual coupling on the terminal voltage vector $\mathbf{V}$ is removed and coupling-free voltage vector $\mathbf{U}$ is found by using (2). At SNR $=40 \mathrm{~dB}$, white gaussian noise is added to coupling-free terminal voltage. The covariance matrix is found by (4), and MUSIC algorithm [20] is used to estimate
TABLe 1: Ground material parameters used in Figure 2 [16].

\begin{tabular}{lcc}
\hline Material & Relative permittivity $\epsilon_{r}$ & Conductivity $\sigma(\mathrm{S} / \mathrm{m})$ \\
\hline Poor ground & 4 & 0.001 \\
Typical ground & 15 & 0.005 \\
Good ground & 25 & 0.02 \\
Sea water & 81 & 5.0 \\
Fresh water & 81 & 0.001 \\
Copper & 1 & $10^{7}$
\end{tabular}

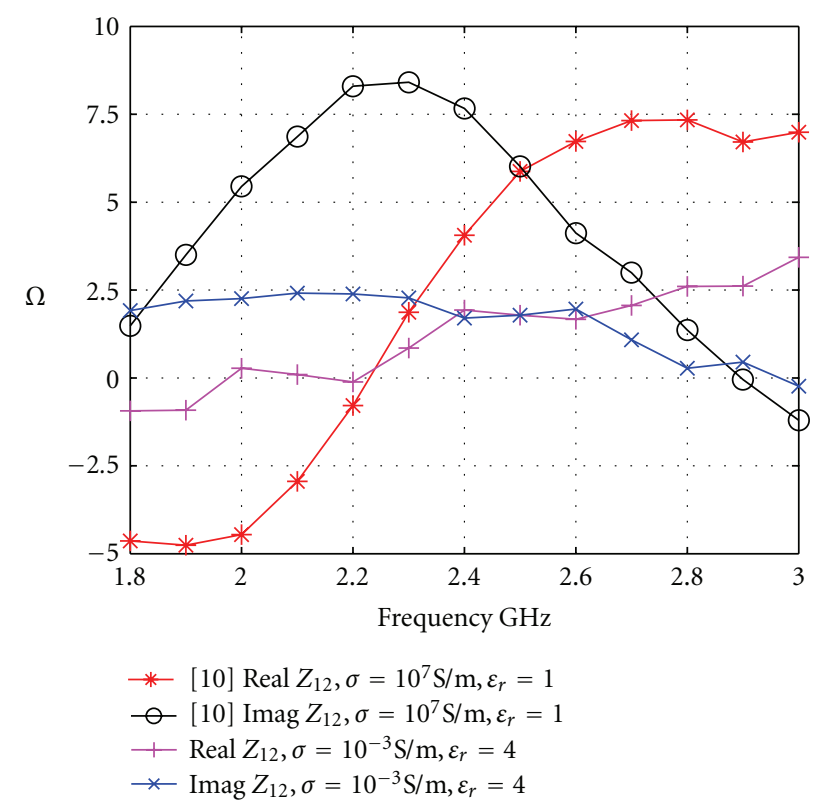

Figure 2: Mutual coupling between two $\lambda / 4$ monopoles over poor ground and with perfect ground [10].

azimuth $(\phi)$ of the incident source. Root mean square error (RMSE) for 1000 Monte Carlo simulations is calculated each for poor and perfect ground conditions.

Table 3 shows RMSE in DOA estimation for three different cases. Case no. 1 shows the RMSE when the antenna is over perfect ground and we remove mutual coupling effects by using mutual impedance matrix measured over the same ground condition. The RMSE is fairly low, which supports the applicability of the method presented in [21]. The critical situation arises when the antenna is placed over a poor ground and the mutual impedance matrix is preestimated over a perfect ground. Now, if we try to remove mutual coupling effects from the measured terminal voltages by using this preestimated mutual impedance matrix according to (2), the result worsens as shown by higher RMSE for case no. 2. The significant increase in RMSE shows that the mutual impedance matrix estimated in [18] over perfect ground is not able to sufficiently remove errors in DOA estimation for the case when the antenna is placed over poor ground. The obvious solution to this problem is to estimate actual mutual impedances over poor ground as shown in the fourth column of Table 2, then use it as in (2) for DOA estimation. The resultant RMSE, (case no. 3 ), is very close to the result found 
TABLE 2: Mutual impedance for five element monopole arrays over perfect and poor ground at $2.4 \mathrm{GHz}$.

\begin{tabular}{lccr}
\hline Mutual impedance component & Perfect gnd [18] & Perfect gnd (FEM) & \multicolumn{2}{c}{ Poor gnd (FEM) } \\
& $\Omega$ & \multicolumn{1}{c}{$\Omega$} & $\Omega$ \\
\hline$Z_{12}=Z_{21}=Z_{23}=Z_{32}=Z_{34}=Z_{43}=Z_{45}=Z_{54}$ & $4.0+j 8.7$ & $-1.3-j 5.6$ & $1.4+j 2.0$ \\
$Z_{13}=Z_{31}=Z_{24}=Z_{42}=Z_{35}=Z_{53}$ & $-1.3-j 5.2$ & $0.8+j 2.7$ & $0.3-j 1.1$ \\
$Z_{14}=Z_{41}=Z_{25}=Z_{52}$ & $0.7+j 3.6$ & $-0.8-j 2.0$ & $-0.03+j 0.13$ \\
$Z_{15}=Z_{51}$ & $-0.4-j 2.7$ & $0.07-j 0.27$ \\
\hline
\end{tabular}

TABLE 3: RMSE of DOA Estimation for array for perfect and poor ground.

\begin{tabular}{lccc}
\hline Case no. & Type of ground for DOA estimation & Type of ground for $\mathbf{Z}$ estimation & RMSE degrees \\
\hline 1 & Perfect ground & Perfect ground/copper & 0.01 \\
2 & Poor ground & Perfect ground/copper & 1.6 \\
3 & Poor ground & Poor ground & 0.03 \\
\hline
\end{tabular}

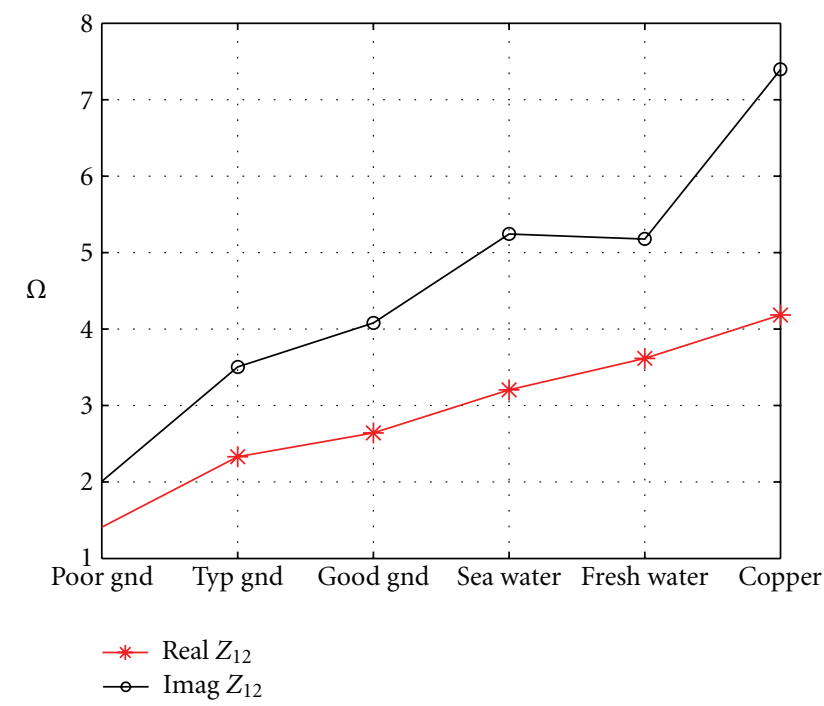

FIGURE 3: Mutual coupling between two $\lambda / 4$ monopoles for various ground types at $2.4 \mathrm{GHz}$.

for the case no. 1 where the antenna placement for DOA estimation and $\mathbf{Z}$ matrix calculation, both was carried out for perfect ground. The argument here is that mobile or portable antennas encounter a variety of ground situations and in these scenarios the preestimated mutual impedance matrix for an ideal case of perfect ground cannot be used to fully remove the errors in DOA estimation. Thus, if the situation permits, one should measure the actual mutual impedance values for the given ground condition before estimating DOAs. However, a universal solution to this problem that works equally well for all ground conditions is still an open problem.

\section{Conclusion}

This research investigated the effects of ground parameters on mutual impedance for DOA estimation. To the best of authors' knowledge, these results are novel and extend the application of an existing technique [10] to real-earth

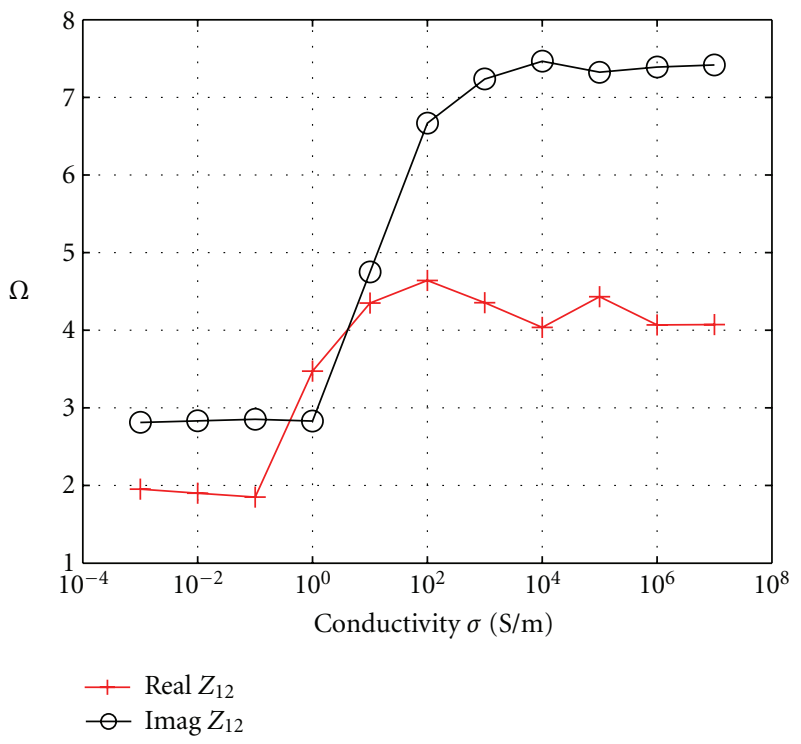

Figure 4: Mutual coupling between two $\lambda / 4$ monopoles at $2.4 \mathrm{GHz}$ for $\sigma=10^{-3}$ to $10^{7} \mathrm{~S} / \mathrm{m}, \epsilon_{r}=10$.

situations. Significant impact of ground constituent parameters on mutual impedance is observed. As a rule of thumb, we suggest to reduce the values of mutual impedance between two vertical monopoles to $50 \%$ of the values found in the anechoic chamber over perfect ground for applications on real earth. The increase of RMSE in case when the antenna is placed over poor ground and preestimated mutual impedance matrix (assuming perfect ground) is used to remove errors in DOA, underscores the importance of this research. We propose that the technique of estimating mutual coupling presented in [10] is applicable to all ground conditions, provided the antennas be placed on respective grounds. It is understood that it is not always possible to pre-estimate mutual impedance for a variety of ground conditions. It is also worth noting that most of the DOA estimation techniques in the literature assume that the array is in free space. Therefore, this research also motivates 


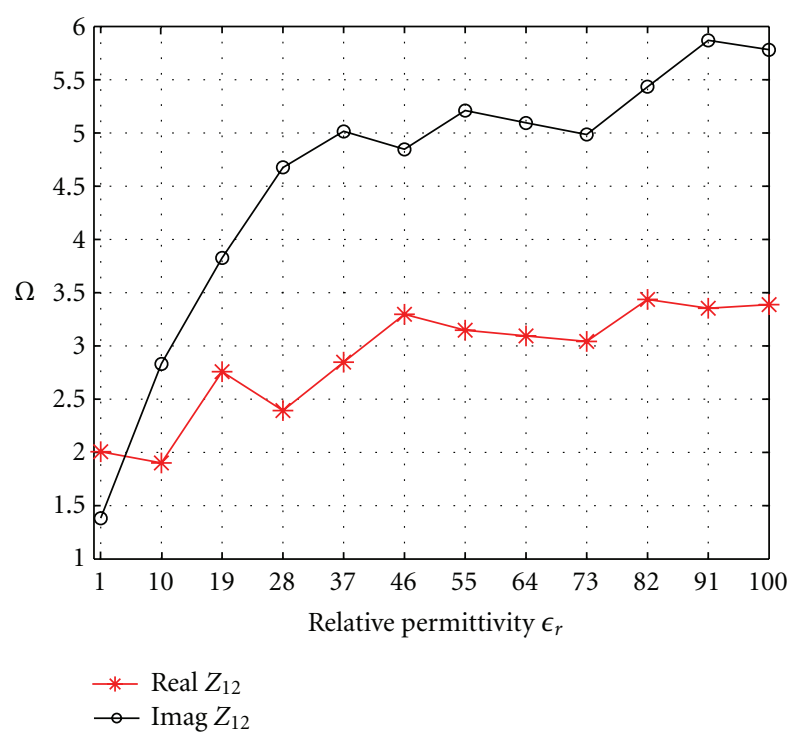

Figure 5: Mutual coupling between two $\lambda / 4$ monopoles at $2.4 \mathrm{GHz}$ for $\epsilon_{r}=1$ to $100, \sigma=0.02 \mathrm{~S} / \mathrm{m}$.

the need for a universal solution of this issue that can be used for any arbitrary ground condition.

The authors wish to continue this work to find effects of ground parameters on other commonly used antenna elements, when they are placed near ground.

\section{References}

[1] H. Krim and M. Viberg, "Two decades of array signal processing research: the parametric approach," IEEE Signal Processing Magazine, vol. 13, no. 4, pp. 67-94, 1996.

[2] B. Friedlander and A. J. Weiss, "Direction finding in the presence of mutual coupling," IEEE Transactions on Antennas and Propagation, vol. 39, no. 3, pp. 273-284, 1991.

[3] F. Sellone and A. Serra, "A novel online mutual coupling compensation algorithm for uniform and linear arrays," IEEE Transactions on Signal Processing, vol. 55, no. 2, pp. 560-573, 2007.

[4] Y. Zhongfu, D. Jisheng, X. Xu, and W. Xiaopei, "DOA estimation for uniform linear array with mutual coupling," IEEE Transactions on Aerospace and Electronic Systems, vol. 45, no. 1, pp. 280-288, 2009.

[5] I. J. Gupta and A. A. Ksienski, "Effect of mutual coupling on the performance of adaptive arrays," IEEE Transactions on Antennas and Propagation, vol. 31, no. 5, pp. 785-791, 1983.

[6] T. Su, K. Dandekar, and H. Ling, "Simulation of mutual coupling effect in circular arrays for direction-finding applications," Microwave and Optical Technology Letters, vol. 26, no. 5, pp. 331-336, 2000.

[7] K. R. Dandekar, L. Hao, and X. Guanghan, "Experimental study of mutual coupling compensation in smart antenna applications," IEEE Transactions on Wireless Communications, vol. 1, no. 3, pp. 480-487, 2002.

[8] Y. Bin, Y. Chengyou, and H. Ye, "Calibration method for mutual coupling between elements based on parallel genetic algorithm," in Proceedings of the 6th World Congress on Intelligent Control and Automation (WCICA '06), vol. 1, pp. 3490-3493, June 2006.
[9] H. Steyskal and J. S. Herd, "Mutual coupling compensation in small array antennas," IEEE Transactions on Antennas and Propagation, vol. 38, no. 12, pp. 1971-1975, 1990.

[10] H. T. Hui, "A new definition of mutual impedance for application in dipole receiving antenna arrays," IEEE Antennas and Wireless Propagation Letters, vol. 3, no. 1, pp. 364-367, 2004.

[11] A. Mirkamali, J. Nateghi, and H. Hadidian, "Evaluation of array calibration methods for DOA estimation in presence of ground, mutual coupling and near zone objects," in Proceedings of the 4th European Conference on Antennas and Propagation (EuCAP '10), pp. 1-4, April 2010.

[12] J. R. Wait, Antenna Theory Part II, vol. 7 of Inter-University Electronics Series, edited by R. E.Collin and F. J. Zucker, McGraw-Hill, New York, NY, USA, 1969.

[13] E. K. Miller, A. J. Poggio, G. J. Burke, and E. S. Selden, "Analysis of wire antennas in the presence of a conducting half-space. part I. the vertical antenna in free space," Canadian Journal of Physics, vol. 50, no. 9, pp. 879-888, 1972.

[14] T. Sarkar, "Analysis of arbitrarily oriented thin wire antenna arrays over imperfect ground planes," Archiv fr Elektronik und Bertragungstechnik, vol. 31, pp. 449-457, 1977.

[15] C. Balanis, Antenna Theory: Analysis and Design, John Wiley \& Sons, New York, NY, USA, 2nd edition, 1997.

[16] T. Rappaport, Wireless Communications: Principles and Practice, Prentice Hall Communications Engineering and Emerging Technologies Series, Prentice Hall, Upper Saddle River, NJ, USA, 2002.

[17] J. D. Jackson, Classical Electrodynamics, John Wiley \& Sons, New York, NY, USA, 3rd edition, 1999.

[18] T. T. Zhang, H. T. Hui, and Y. L. Lu, "Compensation for the mutual coupling effect in the ESPRIT direction finding algorithm by using a more effective method," IEEE Transactions on Antennas and Propagation, vol. 53, no. 4, pp. 1552-1555, 2005.

[19] "Comsol multiphysics modelling and engineering simulation software," 2011, http://www.comsol.com/products/rf/.

[20] R. O. Schmidt, "Multiple emitter location and signal parameter estimation," IEEE Transactions on Antennas and Propagation, vol. 34, no. 3, pp. 276-280, 1986.

[21] H. T. Hui, "A practical approach to compensate for the mutual coupling effect in an adaptive dipole array," IEEE Transactions on Antennas and Propagation, vol. 52, no. 5, pp. 1262-1269, 2004. 

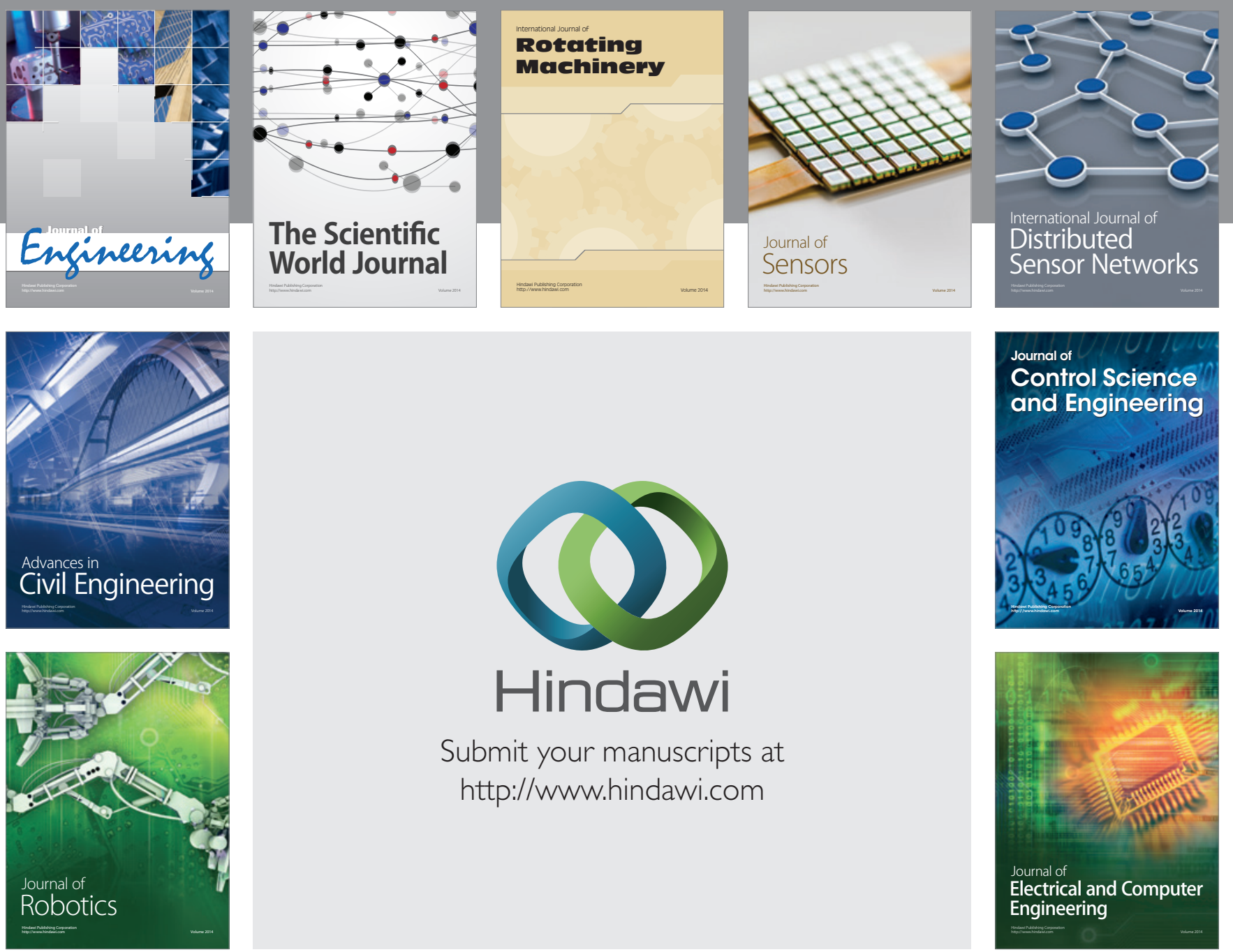

Submit your manuscripts at

http://www.hindawi.com
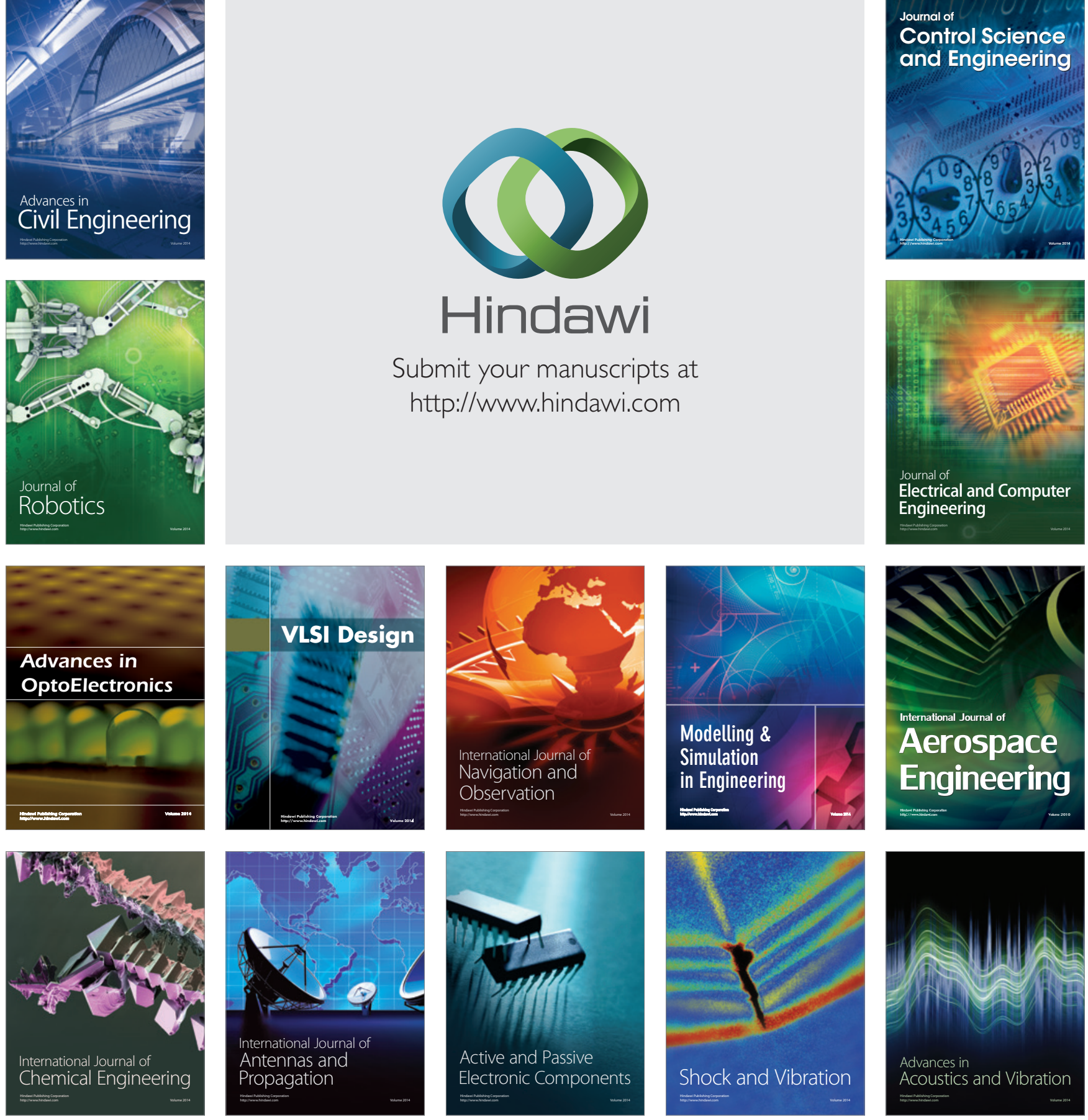